Case Report

\title{
Investigation Report of cVDPV2 Outbreak in Bokh Woreda of Dollo Zone, Somali Regional State, Ethiopia
}

\author{
Diriba Sufa ${ }^{1}$ and Urge Gerema $\mathbb{i}^{2}$ \\ ${ }^{1}$ Ethiopian Public Health Institute (EPHI), Center for Public Health Emergency Management (cPHEM), Addis Ababa, Ethiopia \\ ${ }^{2}$ Department of Biomedical Sciences, Division of Anatomy, College of Medical Sciences, Institute of Health Sciences, \\ Jimma University, Jimma, Ethiopia
}

Correspondence should be addressed to Urge Gerema; urgegerema@gmail.com

Received 29 February 2020; Revised 27 July 2020; Accepted 20 August 2020; Published 26 August 2020

Academic Editor: Gernot Walder

Copyright (C) 2020 Diriba Sufa and Urge Gerema. This is an open access article distributed under the Creative Commons Attribution License, which permits unrestricted use, distribution, and reproduction in any medium, provided the original work is properly cited.

\begin{abstract}
Background. Poliovirus isolates detected in persons or in the environment can fall into three major categories: wild, Sabin and Sabin-like, or vaccine-derived. Detection of wild or vaccine-derived poliovirus may constitute an emergency, which can be categorized as an event that can lead to an outbreak, depending on characteristics of the isolate and the context in which it appears. The aim of the study was investigation report of cVDPV2 outbreak in Bokh woreda of Dollo Zone, Somali regional state, Ethiopia. Methods. A team of experts drawn from different organizations was deployed to Bokh woreda to make detailed field investigation from May 25 to June 17, 2019. By using standard World Health Organization polio outbreak investigation checklist, document review of surveillance, immunization, and clinical data related to the case was made. Key informant's interview was made to health professionals, managers, parents of case, woreda and kebele leaders, religious leaders, and HEWs related to acute flaccid paralysis outbreak. Result. The notified AFP case was a 39-month-old female from Angalo kebele of Bokh woreda, Dollo Zone. On 19th May 2019, the patient developed high grade fever and was taken to Angalo Health Post on 20th May 2019. As per the examination by a health extension worker, the child had high grade fever and neck stiffness with preliminary diagnosis of meningitis for which ceftriaxone injection was prescribed. Contact sample was taken from three children on 28th May 2019 and 29th May 2019 and was sent to Addis Ababa National Polio Laboratory. All contact stool samples were found to be positive for poliovirus type 2 and referred for sequencing in National Institute of Communicable Diseases (NICD), South Africa, the Regional Polio Reference Laboratory. Conclusion and Recommendation. The clinical presentation of the cases is compatible with poliovirus infection, improving the quality and coverage of supplementary polio immunization activities through proper planning; strict supervision and follow-up can reduce the occurrence of acute flaccid paralysis.
\end{abstract}

\section{Introduction}

Poliovirus isolates detected in persons or in the environment can fall into three major categories: wild, Sabin and Sabinlike, or vaccine-derived [1-3]. Detection of wild or vaccinederived poliovirus may constitute an emergency, which can be categorized as an event that can lead to an outbreak depending on characteristics of the isolate and the context in which it appears $[2,4-6]$. Genetically, vaccine-derived poliovirus (VDPV) strains can emerge during vaccine use and spread in underimmunized populations, becoming circulating (cVDPV) strains and resulting in outbreaks of paralytic poliomyelitis [2, 7-10]. The global polio eradication was set up in 1988 when the World Health Assembly (WHA) passed a resolution to eradicate polio by the year 2000 $[11,12]$. Due to many challenges, the goal set for 2000 was not met [12]. The global polio eradication initiatives developed the polio eradication and endgame strategic plan covering the period 2013 to 2018 , aiming at a polio-free world by 2018 [13, 14]. The basic strategies for eradicating polio include the following: (i) immunization of every child aged less than one year with at least three doses of oral polio vaccine (OPV); (ii) national immunization days (NIDS) in which all children less than five years of age receive extra 
doses of OPV on two days separated by at least 28 days; (iii) surveillance for acute flaccid paralysis to identify all reservoirs of wild poliovirus transmission; and (iv) extensive house to house immunization mopping-up campaigns in the final stages in areas where wild poliovirus transmission persists $[12,15,16]$. In the prepolio eradication era, polio was generally endemic in many African countries with 1597 polio cases recorded in the continent in 1995 [17].

The implementation of the polio eradication strategies in Ethiopia started in 1996 [18]. Ethiopia was polio free for four years from January 2001 until December 2004. From December 2004 to February 2006, a total of 24 cases were confirmed from Tigray, Amhara, and Oromia regional states. In 2004, the surveillance performance for acute flaccid paralysis (AFP) at national level was above the targets as of February 2005 [17, 19]. We present the AFP cases from Angalo kebele of Bokh woreda, Dollo Zone, which is above the targets.

1.1. Immunization Service Status in the Woreda. Bokh woreda provides routine immunization service both by static and outreach strategies [20]. The trend of the RI coverage for the woreda showed rapid decline over the last four years. Data obtained from the Regional Health Bureau showed that the average SIA coverage of the woreda was $98 \%, 97 \%$, $97.5 \%$, and $97 \%$ in the year 2015, 2016, 2017, and 2019, respectively. Only five $(16.7 \%)$ of under-five children reported to have received at least 3 doses of OPV during all rounds of polio SIAs [20]. The aim of the study was to present investigation report of cVDPV2 outbreak in Bokh woreda of Dollo Zone, Somali regional state, Ethiopia.

1.2. Case Presentation. The notified AFP case is a 39-monthold female from Angalo kebele of Bokh woreda, Dollo Zone. On 19th May 2019, the child developed high grade fever and was taken to Angalo Health Post on 20th May 2019. As per the examination by health extension worker, the child had high grade fever and neck stiffness with preliminary diagnosis of meningitis for which ceftriaxone injection was prescribed. Despite the administration of antibiotic injection for two days, the child become critically sick and started to have paralysis of the lower limb followed by weakness of the upper extremities. The case was then taken to Buhodle, Somaliland, for further diagnosis and management and notified as an AFP case from Somalia on 25th May 2019. Stool sample was obtained and taken to Nairobi, Kenya, on 27th May 2019. On 9th June 2019, it was reported, through World Health Organization (WHO), the laboratory results of the AFP case turned out to be positive for poliovirus-2. The WHO Somaliland polio team at Buhodle was notified about the case on 25th May 2019 and investigation started on 26th May 2019. The first and second stool samples were collected on 27th May 2019 and 28th May 2019, respectively, and sent to polio laboratory at Kenya Medical Research Institute (KEMRI) in Nairobi for laboratory investigation.

Contact sample was taken from three children on 28th May 2019 and 29th May 2019 and was sent to Addis Ababa National Polio Laboratory. All contact stool samples were found to be positive for poliovirus type 2 and referred for sequencing in National Institute of Communicable Diseases (NICD), South Africa, the Regional Polio Reference Lab. The ages of these close contacts are 56 months, 54 months, and 33 months. Of three contacts, only one child received one dose of OPV through RI. All the three contacts got one dose of OPV through SIA according to information from mothers of the children.

\section{Discussion}

The case here described represents cVDPV2 outbreak. cVDPV2 was clinically suspected because of the appearance of child, high grade fever, neck stiffness, and paralysis of the lower limb followed by weakness of the upper extremities. It was confirmed with isolation of CVDPV in stool specimens collected from the suspected case or from a close contact.

Global experiences showed that VDPV has shown increased trends in areas with low immunization coverage of both routine and SIA [11]. The incidence of type-2 strain of the VDPV is disproportionally higher than the other types [20]. Ethiopia switched providing tOPV as routine since March 2016 following eradication of type poliovirus from the world. Since then, a number of SIAs has been conducted in Ethiopia [21]. Absence of routine OPV vaccination and repeated uses of bOPV might have contributed to occurrence of VDPV in this case. Though family and community have positive attitudes towards and high demands for immunization, the services are not reaching them. Apart from population mobility, unreliable security, transport, and manpower shortages, lack of proper planning contributed to low immunization coverage.

Case detection and reporting trends are optimal. Though knowledge gaps exist among health workers at all levels on other causes of AFP, there are no unreported cases found. No missed cases were also found during house-to-house case searches and from in-depth inquiry of family and communities. It is worth-mentioning that community and its leaders are well aware of the signs and symptoms of the disease and are highly sensitive for reporting to the health system. The case was immediately taken to nearby health center. Two of the contact cases from household and neighborhood were not vaccinated by routine immunization. All contacts received one OPV dose from 2017 SIAs. The lab results of all contacts revealed to be positive for poliovirus-type two at the national polio laboratory and were sent for further investigation to South Africa. The newly found AFP cases have also not received any dose from RI and received SIA doses. It was also noted that there was no significant travel history to and from other woredas of the region and neighboring Somalia. The results of lab tests for both cases are pending at EPHI.

\section{Conclusion}

Based on the detailed case assessment, epidemiological, sociodemographic, health status including EPI, and surveillance investigation and laboratory findings, the investigation team comes up with the following conclusion. 
As per the information collected from the parents and the locals, the family of the index case has been living in the area for more than seven years and has lived within the same kebele prior, which ensures the child is from Ethiopian side. The clinical presentation of the case is compatible with poliovirus infection.

\section{Abbreviations}

$\begin{array}{ll}\text { AFP: } & \text { Acute flaccid paralysis } \\ \text { bOPV: } & \text { Bivalent oral polio vaccine } \\ \text { CBS: } & \text { Community-based surveillance } \\ \text { cPHEM: } & \text { Circulating Public Health Emergency } \\ & \text { Management } \\ \text { CVDPV2: } & \text { Circulating vaccine-derived poliovirus } \\ \text { EPHI: } & \text { Ethiopian Public Health Institute } \\ \text { EPI: } & \text { Expanded program immunization } \\ \text { FGD: } & \text { Focused group discussion } \\ \text { FMOH: } & \text { Federal Ministry of Health } \\ \text { IDSR: } & \text { Integrated Disease Surveillance and Response } \\ \text { KEMRI: } & \text { Kenya Medical Research Institute } \\ \text { NIDS: } & \text { National immunization days } \\ \text { OPV: } & \text { Oral polio vaccine } \\ \text { PHEM: } & \text { Public Health Emergency Management } \\ \text { PV2: } & \text { Polio vaccine } 2 \\ \text { RHB: } & \text { Regional Health Bureau } \\ \text { RI: } & \text { Routine immunization } \\ \text { SIA: } & \text { Supplementary polio immunizations activities } \\ \text { tOPV: } & \text { Trivalent oral polio vaccine } \\ \text { UNICEF: } & \text { United Nations Children's Fund } \\ \text { VDPV: } & \text { Vaccine-derived poliovirus } \\ \text { WHO: } & \text { World Health Organization. }\end{array}$

\section{Data Availability}

The datasets used and/or analyzed during the current study are available from the corresponding author on reasonable request.

\section{Consent}

Informed oral consent was obtained from each family subject, and permission was obtained for publication.

\section{Conflicts of Interest}

The authors declare that there are no conflicts of interest.

\section{Authors' Contributions}

Diriba Sufa and Urge Gerema were involved in writing the manuscript and managing the overall progress of the study. Both authors read and approved the final manuscripts that could inappropriately influence or bias the content of the paper.

\section{Acknowledgments}

The authors would like to thank all government $(\mathrm{FMOH}$, $\mathrm{RHB}, \mathrm{ZOH}$, and $\mathrm{WHB}$ ) and partner organizations (WHO,
UNICEF, EPHI, USAID, and Kenya Medical Research Institute) for commitment and timely action to investigate and produce evidences for action and further improvement. The authors extend their thanks to Dollo Zone administration, the community of the Bokh woreda, case and her family, woreda administration, and all supporting staffs including drivers for their commitment and cooperation during this investigation. Finally, the authors would like to extend their heartfelt thanks to all health extension workers and health professionals especially laboratory technologist who diagnose and manage the case in general.

\section{References}

[1] K. M. O’Reilly, R. Verity, E Durry et al., "Population sensitivity of acute flaccid paralysis and environmental surveillance for serotype 1 poliovirus in Pakistan: an observational study," BMC Infectious Diseases, vol. 18, no. 1, p. 176, 2018.

[2] D. A. Kalkowska, R. J. Tebbens, M. A. Pallansch, S. L. Cochi, S. G. Wassilak, and K. M. Thompson, "Modeling undetected live poliovirus circulation after apparent interruption of transmission: implications for surveillance and vaccination," BMC Infectious Diseases, vol. 15, no. 1, p. 66, December 2015.

[3] A. F. Polio, Ghana Weekly Epidemiological Report, 2019.

[4] Global Polio Eradication Initiative, Standard Operating Procedures: Responding to a Poliovirus Event or Outbreak, World Health Organization, Global Polio Eradication Initiative, Geneva, Switzerland, 2017.

[5] I. M. Blake, M. Pons-Salort, N. A. Molodecky et al., “Type 2 poliovirus detection after global withdrawal of trivalent oral vaccine," New England Journal of Medicine, vol. 379, no. 9, pp. 834-845, 2018

[6] T. Foiadelli, S. Savasta, A Battistone et al., "Nucleotide variation in Sabin type 3 poliovirus from an Albanian infant with agammaglobulinemia and vaccine associated poliomyelitis," BMC Infectious Diseases, vol. 16, no. 1, p. 277, 2016.

[7] J. Jorba, O. M. Diop, J. Iber et al., "Update on vaccine-derived poliovirus outbreaks - worldwide, january 2018-june 2019," MMWR. Morbidity and Mortality Weekly Report, vol. 68, no. 45, p. 1024, 2019.

[8] S. J. Kim, S. H. Kim, Y. M. Jee, and J. S. Kim, "Vaccine-associated paralytic poliomyelitis: a case report of flaccid monoparesis after oral polio vaccine," Journal of Korean Medical Science, vol. 22, no. 2, pp. 362-364, 2007.

[9] M. M. Alleman, R. Chitale, C. C Burns et al., "Vaccine-derived poliovirus outbreaks and events-three provinces, democratic republic of the Congo," Morbidity and Mortality Weekly Report, vol. 67, no. 10, p. 300, 2018.

[10] C. Mbaeyi, M. M. Alleman, D. Ehrhardt et al., "Update on vaccine-derived poliovirus outbreaks-democratic republic of the Congo and horn of Africa, 2017-2018," MMWR. Morbidity and Mortality Weekly Report, vol. 68, no. 9, p. 225, 2019.

[11] Global Polio Eradication Initiative and World Health Organization, Global Polio Eradication Initiative: Estimated External Financial Resource Requirements 2002-2005, as of 1 September 2001, pp. 11-14, World Health Organization, Geneva, Switzerland, 2001.

[12] S. L. Cochi, L. Hegg, A. Kaur, C. Pandak, and H. Jafari, "The global polio eradication initiative: progress, lessons learned, and polio legacy transition planning," Health Affairs, vol. 35, no. 2, pp. 277-283, 2016.

[13] C. Mbaeyi, Z. M. Wadood, and T. Moran, "Strategic response to an outbreak of circulating vaccine-derived poliovirus type 
2-Syria," Morbidity and Mortality Weekly Report, vol. 67, no. 24, p. 690, 2018.

[14] S. L. Cochi, L. Hegg, A. Kaur, C. Pandak, and H. Jafari, "The global polio eradication initiative: progress, lessons learned, and polio legacy transition planning," Health Affairs, vol. 35, no. 2, pp. 277-283, 2016.

[15] S. Machingaidze, C. S. Wiysonge, and G. D. Hussey, "Strengthening the expanded programme on immunization in Africa: looking beyond 2015," PLoS Med, vol. 10, no. 3, Article ID e1001405, 2013.

[16] T. H. Ndlovu, L. Fernandes, and R. J. Burnett, "Expanded programme on immunisation: coverage of 12-23 months-olds and reasons for non-vaccination in muldersdrift (krugersdorp), south africa," Doctoral Dissertation, University of Limpopo, University of Limpopo, Polokwane, South Africa, , 2019.

[17] G. Mesfin, W. Schluter, A Gebremariam et al., "Polio outbreak response in Ethiopia," East African Medical Journal, vol. 85, no. 5, pp. 222-231, 2008.

[18] World Health Organization, Report of the thirty-second session of the Regional Committee for the Eastern Mediterranean, World Health Organization, Geneva, Switzerland, 1985.

[19] H. Belete, T. Kidane, F. Bisrat, M. Molla, S. Mounier-Jack, and Y. Kitaw, "Routine immunization in Ethiopia," The Ethiopian Journal of Health Development (EJHD), vol. 29, no. 1, 2015.

[20] B. Woreda of Dollo Zone, Somali Regional State Health Office Report, 2018.

[21] A. A. Tegegne, F. Braka, M. E Shebeshi et al., "Characteristics of wild polio virus outbreak investigation and response in Ethiopia in 2013-2014: implications for prevention of outbreaks due to importations," BMC Infectious Diseases, vol. 18, no. 1, pp. 1-0, 2018. 